\title{
Communicating with our Metastatic Breast Cancer Patients about the Goals of Treatment and Goals of Palliative Care
}

\author{
Lillie D. Shockney* \\ Department of Surgery and Oncology, Johns Hopkins Breast Center, USA
}

Received: November 28, 2013; Accepted: November 31, 2013; Published: Jaunary 07, 2014

"Corresponding author: Lillie D. Shockney, University Distinguished Service Associate Professor of Breast Cancer, Department of Surgery and Oncology, Administrative Director, the Johns Hopkins Breast Center, 600 N Wolfe Street, Carnegie, Room 683, Baltimore, MD 21287, USA, Tel: 410-614-2853; Fax: 443-873-5014; E-mail: shockli@jhmi.edu

\section{Editorial}

All of us as clinicians want to provide a glimmer of hope to our patients battling advanced metastatic breast cancer. Initially we may be able to confidently talk about a stage IV breast cancer patient likely living quite a few years on hormonal therapy as a means to keep her disease in control. At some point however, the disease does eventually progress and decisions need to be made about what treatments next to consider. These treatments we also know will be more toxic and riddled with side effects that will likely impact the patient's quality of life.

When caring for patients with curative intent having been diagnosed with stage I - III breast cancer, we talk to the patient about the pros and cons, risks and benefits, side effects, and goals of the treatment. We are able to speak confidently in mathematical percentages of how likely is the cancer to recur and what reduction in this risk will this treatment regimen afford the patient.

When speaking to someone with stage IV breast cancer however, we may not be as comfortable discussing such statistics. And the patient may be hesitant to ask. The people on both sides of this conversation are buffering on behalf of the other as to not say too much or learn too much about the purpose of the treatment and the likelihood that it will be of benefit at all. Physicians however feel the need to offer treatments even if the chance of them helping the patient is very unlikely and the patients feel the need to continue treatment to please the doctor as well as calm the anxieties of their family members by still demonstrating they are trying to get a miraculous cure.

I conduct retreats for patients and their spouses battling metastatic breast cancer. I always ask this question: How (not who but HOW) will the decision be made when it is time to stop treatment? The responses are always the same- my doctor will let me know. On the flip side of this equation is the doctor's response to that same question when focused groups have been held for that purpose. The oncologists say-my patient will let me know. This is a life a bad marriage. People mean well but the outcome is not what is really desired. And that patient only has one life. She can't redo the decisions that have been made for her by her treating physicians either. She has one opportunity to do this right.

It is time to have a more candid discussion with our patients about palliative care being incorporated early on while treatment is still actively taking place as well as discuss the goals of care. (Note I did not say goals of treatment.) We should begin with really knowing our patient well beyond her pathology. Is she married, have young children, work outside of the home. What gives her joy (or gave her joy before she became ill)? What were her life goals too before her diagnosis of this life limiting disease? Are there specific lives goals she is hoping to achieve that are short term (for example, seeing her daughter graduate from high school in 2 months)? Based on our own experience with this disease and its likely time frame of progression, are there goals she is telling us that are likely unrealistic (for example, seeing her daughter graduate from high school in 10 years)?

\section{A patient story that provide insight into this issue \\ "I don't want to live the remainder of my life with you."--}

A patient had been battling metastatic breast cancer for 8 years. All approved treatments had now been exhausted and she was directed to another facility to consider a phase I clinical trial. The drive there was nearly 2 hours due to the known congested traffic that happens all day, every day. She met with a resident who explained the protocol-IV therapy 5 days a week. Time needed to do the infusions each day would be 4 hours. She would need someone to bring her and take her home due to the GI side effects that are very likely to occur. She would also need to come on Saturday mornings for blood work. The benefit would be the possibility of extending her life while she received the treatment and she would remain on the treatment as long as the disease appeared to not be progressing further. Other patient participating on this clinical trial so far was lasting on the treatments for 2-4 months. She listened to all of this. She then told the doctor that she was going to decline going on this phase I clinical trial. The resident said to her that he didn't understand her rationale for declining enrollment given "this was her last 
resort at any kind of treatment." Her response was as follows: "It would take me 2 hours to get here and 4 hours while here then 2 more hours to get home, all the while I am dealing with nausea and vomiting. My husband would likely be the person driving me so he would be off from work for an undefined period of time, maybe months. When I would get home I still would have nausea. I would need to be doing this every day Monday through Friday as well as returning here again on Saturday. Frankly, I don't want to spend the rest of my life with you. I will spend my remaining time at home with my husband, daughter and new grandson."

Her focus was clearly on quality of life as it should have been. She survived 4 more months and experienced good quality of life with pain in control, affairs in order, devoted time with her family, and well prepared for end of life. This care (not treatment), which was delivered by Hospice at home and her medical oncologist from Johns Hopkins (who made house calls on the weekends to her home) provided her exactly what she needed - quality time spent with those who gave her joy. It also likely provided her quantity of time since she was not on toxic drugs that would likely have hastened her death.

How do we engage in such a conversation with our patients, however, so as to discuss what is important to them? We need to incorporate such communication training into medical school educational programs. For those already in active practice that are not familiar or comfortable with having such discussions, consider accompanying a palliative care medical oncologist on their rounds in the hospital as well as in their clinic to witness how these discussions happen. Begin with patients who you are not involved with then advance into accompanying this specialist when you make a referral on behalf of your own patients.

Treatment for treatment's sake should never be our goal or a goal we require a patient to fulfill for us. We need to put the survival curve charts down and focus on the more important measurement-quality of life for our patients. We need to measure quality of life based on how the patient deems it to be measured and not assuming that based on our observation we can confirm that their quality of life is good.

So when you are meeting with your next patient who has gotten the news that she has advanced metastatic breast cancer, consider taking a different tact. Get to know her and her family at the time of that first consultation. As time progresses and decisions are jointly made with the patient weighing in on what are the appropriate next best steps for her to be taking, you will find yourself providing care that is truly better than it ever was before. You will be helping to set the stage for her eventually to experience a good death, pain free, leaving a legacy (which doesn't mean money), finding and acknowledging her purpose of having lived, and dying knowing that she will be remembered in a wonderful way. This is how we as clinicians should be measuredhow we accomplish this end of life goals for our patients. 